\title{
A REVIEW OF THE EVIDENCE FOR A "GULF WAR SYNDROME"
}

Khalida Ismail

$\mathrm{O}$ n 2 August 1990, Iraq invaded Kuwait. Four days later, nearly 700000 US troops and an international coalition of 100000 military personnel were mobilised to the Gulf under Operation Desert Shield, which included 53000 members of the UK Armed Forces under Operation Granby. The air campaign, Operation Desert Storm, began on the 17 January 1991. On 24 February 1991, a ground war was conducted which lasted only four days. Thousands of Iraqi soldiers were killed in the hostilities, on the infamous Basra "Death" Road, one of the main routes they used to enter and leave Kuwait. There were less than 300 deaths in the allied forces.

Within months after the hostilities had ended reports of US Gulf veterans complaining of various symptoms began emerging. Veterans groups, the media, and certain researchers alleged that some Gulf veterans were suffering from a "Gulf War syndrome", a new disorder, which they believed was caused by various environmental exposures while in the Gulf theatre. This article addresses the case for a "Gulf War syndrome".

The process by which a new illness arises depends first on establishing whether its clinical features are sufficiently different from other known conditions and then whether aetiological factor(s) can be identified. ${ }^{1}$ Social and cultural factors, such as the perception of one's health and causes of disease, are also relevant in the emergence of a new disorder. Outcome, interventions, and lessons for the future are important. The literature on each of these topics will be reviewed.

\section{CLINICAL FEATURES OF ILL HEALTH IN GULF VETERANS}

\section{Epidemiological studies}

Initial case reports described non-specific symptoms such as fatigue, headaches, and rashes that did not fall into recognised diagnostic categories. In the USA, Gulf veterans were invited to join voluntary medical registries run by the US Department of Defense for those still serving (the Comprehensive Clinical Evaluation Program) ${ }^{2}$ and the Department of Veteran Affairs for discharged personnel. A few individuals did have recognised diseases, such as leishmaniasis, but the majority presented with non-specific symptoms. Fatigue, headache, memory problems, sleep disturbances, skin rashes, joint pains, and dyspnoea were the most commonly reported symptoms in both registries. Three International Classification of Diseases, ninth revision (ICD-9) categories, musculoskeletal disorders (18.6\%), mental disorders (18.3\%), and symptoms, signs and ill defined conditions $(17.8 \%$ ), accounted for over $50 \%$ of diagnoses of the first 20000 veterans who participated in the Comprehensive Clinical Evaluation Program. ${ }^{2}$ The UK Ministry of Defence voluntary register, the Medical Assessment Program, found no evidence of a new syndrome nor a dramatic over representation of any one disorder. ${ }^{3}$ As voluntary registers may have been over represented by people who perceive themselves to be ill, studies representative of the military population were needed.

Population based studies have found that Gulf veterans consistently self report a wide range of non-specific symptoms and conditions compared to military controls. A telephone survey of a random sample of Gulf veterans who resided in Iowa when they enlisted had higher rates of self reported medical and psychiatric symptoms, such as asthma, depression, post-traumatic stress disorder, fatigue, fibromyalgia, and alcohol abuse, than contemporary military personnel who were not deployed to the Gulf. ${ }^{4}$ A survey of nearly 4000 currently active personnel in four US Air Force units found that Gulf veterans reported an excess of all 35 symptoms enquired compared to non-Gulf veterans; the most common were sinus congestion, headache, fatigue, joint pain and stiffness, cognitive difficulties, and difficulty sleeping. ${ }^{5}$ In a survey of 1500 active duty US Navy

Correspondence to: Dr Khalida Ismail, Department of Psychological Medicine, Guy's, King's and St Thomas' School of Medicine, 103 Denmark Hill, London SE24 0AQ, UK

khalida.ismail@iop.kcl.ac.uk mobile construction battalion personnel (Seabees), Gulf veterans reported a higher prevalence of symptoms, mainly fatigue, forgetfulness, sleeping difficulties, rash, joint pains and headaches, psychological symptoms consistent with post-traumatic stress disorder, and depression. However, objective assessment did not reveal any differences between hand grip strength, serum ferritin, C-reactive protein, haptoglobin or lung function. ${ }^{6}$ In a survey of military personnel in the UK, Gulf veterans reported all 50 symptoms enquired two to three times more frequently than two 


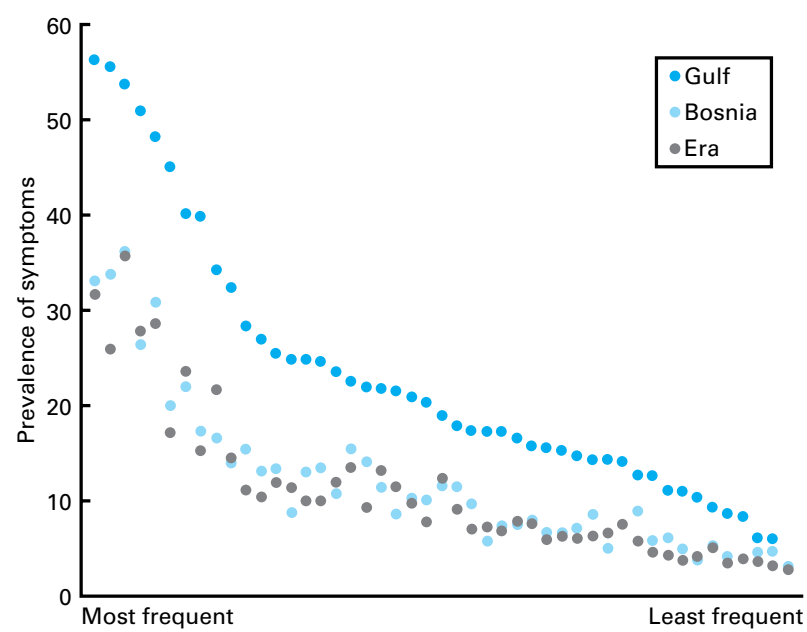

Figure 1 Distribution of 50 symptoms in three UK military cohorts: deployed to the Gulf, deployed to Bosnia, and Era (serving in the military during the Gulf conflict but not deployed to the Gulf).

military controls, veterans who were on another deployment (Bosnia peace keeping), and veterans who were in active service at the time but not deployed to the Gulf (fig 1). The most common symptoms in all three cohorts were sleep difficulties, irritability, headaches, fatigue, forgetfulness, and joint pains (box 1). Symptom prevalence in the control groups was similar to each other suggesting that there was a Gulf specific rather than deployment effect. While the perception of general health was significantly lower in the Gulf group than the other two groups, the level of physical functioning was about the same in all three groups.

\begin{tabular}{|llll|}
\hline $\begin{array}{l}\text { Box 1: The } 15 \text { most frequent symptoms (\%) by } \\
\text { deployment (men) }\end{array}$ & & \\
& & & \\
Symptoms & $\begin{array}{l}\text { Gulf } \\
(n=3284)\end{array}$ & $\begin{array}{l}\text { Bosnia } \\
(n=1815)\end{array}$ & $\begin{array}{l}\text { Era } \\
(n=2408)\end{array}$ \\
\hline Feeling unrefreshed after sleep & 56.1 & 33.0 & 31.6 \\
Irritability/outbursts of anger & 55.2 & 33.6 & 25.8 \\
Headaches & 53.5 & 36.0 & 35.6 \\
Fatigue & 50.7 & 26.3 & 27.7 \\
Sleeping difficulties & 48.0 & 30.7 & 28.4 \\
Forgetfulness & 44.9 & 19.9 & 17.1 \\
Joint stiffness & 40.0 & 21.8 & 23.5 \\
Loss of concentration & 39.7 & 17.2 & 15.1 \\
Flatulence/burping & 34.1 & 16.4 & 21.5 \\
Pain without swelling/redness & 32.2 & 13.8 & 14.4 \\
Feeling distant/cut off from & 28.1 & 15.2 & 11.0 \\
other & & & \\
Avoiding doing & & 13.0 & 10.3 \\
things/situations & 26.8 & 13.2 & 11.8 \\
Chest pain & & 13.3 & 9.8 \\
Feeling jumpy/easily startled & 25.3 & 15.2 & 13.3 \\
Sore throat & 22.3 & & \\
\hline
\end{tabular}

There was no consistent excess of admissions to military hospitals for explained and unexplained conditions among active duty Gulf veterans. ${ }^{8}$ An eight year retrospective study of all UK Gulf veterans reported a slightly increased mortality rate from external causes, mainly accidents, compared to matched non-Gulf military personnel while mortality rates from disease related causes were lower. ${ }^{9}$ These results are similar to earlier US findings involving over 200000 veterans. $^{3}$

\section{Statistical approaches to Gulf war related ill health}

Factor analysis is a set of statistical methods that examine the latent factor structure that underlies the correlations between the set of symptoms observed. It has been used as a

\section{Box 2: Clinical features of "Gulf War syndrome"}

- Gulf veterans are reporting symptoms two to three times more frequently than military personnel who were not deployed to the Gulf

- The symptoms are multisystem and non-specific, such as fatigue, headache, subjective memory problems, sleep disturbance, and musculoskeletal pains

- The underlying pattern of symptom reporting is not unique to Gulf veterans

- There is some evidence that symptoms are associated with psychological conditions

- Despite the increased symptom reporting, the overall physical functioning of Gulf veterans is not greatly impaired

parsimonious method of representing and validating multisymptom data on Gulf War veterans. ${ }^{5}$ One unit in Texas suggested that there was a "Gulf War syndrome" based solely on the factor structure in a non-representative sample of US Gulf veterans. ${ }^{31011}$ This led to much controversy in the scientific community and Gulf veterans particularly after further factor analysis studies were unable to corroborate the findings. ${ }^{10}$ In a factor analysis using 50 symptoms in a UK sample of Gulf veterans, the first three factors were characterised by symptoms of mood cognition, such as headaches, irritability, sleep difficulties, and distressing dreams; respiratory system symptoms, such as shortness of breath and wheezing; and peripheral nervous system symptoms, such as tingling and numbness. There were no great differences in the three factor structures in Gulf veterans compared to Bosnia veterans and non-deployed veterans. ${ }^{10}$ In a US sample of active duty Navy personnel, five factors were identified which were similar in both Gulf and non-Gulf veterans, although Gulf veterans had higher factor scores reflecting greater severity of symptoms. Another US study derived a three factor structure with similar symptoms loading onto them in both Gulf and non-Gulf samples. They labelled these factors as somatic distress, psychological distress, and panic. The factors were highly convergent with each other in each of the two samples. ${ }^{3}$

\section{Relation between symptoms and mental health}

All the voluntary registers and population based studies have consistently shown that psychiatric symptoms ${ }^{4-7}$ and mental disorders, ${ }^{28}$ are more common in Gulf veterans than non-Gulf controls. A significant proportion of veterans on the voluntary register who had received a "symptoms, signs, and ill-defined condition" diagnosis had a psychiatric disorder, either depression, somatoform disorder, tension headaches, or post-traumatic stress disorder. A dose response association between the number of symptoms reported and post-traumatic stress disorder and depression in Gulf veterans has also been reported.

The overall clinical picture to date is that Gulf veterans are reporting more symptoms, which appear to be multi organ and non-specific. The most common symptoms are related to mood and cognitive functioning and appear to differ from other military populations only in frequency and severity. An important limitation of most studies is that they have used self report measures of illness as opposed to objective measures; this increases the risk of recall bias and inadvertent endorsement of symptom in the questionnaires, and may have led to overestimation of health problems. 


\section{Gulf War specific exposures}

A number of environmental factors specific to the Gulf conflict such as suspected chemical weapons, organophosphate pesticides, immunisations, pyridostigmine bromide, oil well fires, and depleted uranium have been postulated as being linked to the syndrome. Several population based studies have reported associations between a wide range of self report exposures and ill health. ${ }^{467}$

Depleted uranium is used in ammunition and armoury. A small group of soldiers were involved in handling depleted uranium which was then excreted, but no health effects have been found; monitoring for late sequelae continues in this sample.

Over 600 oil wells were set alight at the end of the ground war which led to plumes of black clouds. Environmental monitoring studies at the time did not find excess levels of toxic gases such as hydrogen sulfide and sulfur dioxide, but there was an increase in the level of fine particulate matter. Military personnel involved in bringing the fires under control were appropriately protected. ${ }^{11}$ Smoke fire can cause eye and nose irritation and decreased pulmonary function. While respiratory symptoms are commonly reported, the sparse data on objective pulmonary function suggests that there is no objective evidence of respiratory damage attributable to oil well smoke. ${ }^{6}$

There was a real threat of nerve agents, such as sarin, and blister agents, such as mustard, being released by Iraq and chemical weapons alarms were in widespread use. There were frequent, sometimes several times a day, presumed false, alarms and on each occasion personnel were required to put on nuclear-biological-chemical (NBC) suits which were uncomfortable and induced partial sensory deprivation. There was an accidental destruction of an Iraqi arms dump with release of chemical agents at Khamisiyah but no casualties were reported at the time, ${ }^{11}$ and there was no post-war increase in hospitalisations in those who have possibly been exposed. Despite the lack of objective evidence of widespread exposure to chemical weapons, the current state of knowledge is that, although unlikely, there is insufficient evidence to rule out that short periods of sublethal doses of sarin may cause neurological damage. ${ }^{11}$

Pyridostigmine bromide was used as pre-treatment for possible exposure to nerve gas. It acts by inhibiting cholinesterase at the neuromuscular junction; the actual doses, frequency, and duration of pyridostigmine bromide administered varied from unit to unit. Some investigators have suggested that pyridostigmine bromide may be a candidate exposure because some individuals are genetically more susceptible to pyridostigmine bromide and/or it may have acted synergistically with pesticides. ${ }^{12}$ Animal models have suggested that stress may increase blood brain barrier permeability to pyridostigmine bromide, although this has been challenged. Self report pyridostigmine bromide intake was not associated with post war handgrip strength. Pyridostigmine bromide is used in much higher doses for patients with myasthenia gravis which suggests it is unlikely to have been toxic to Gulf veterans.

Varying amounts of pesticides were issued (DEET and premethrin) to combat the desert pests that inhabited the military camps with personnel. While there is usually gross evidence of toxic effects of high doses of organosphophates, the delayed effects of chronic low level exposure, perhaps in conjunction with pyridostigmine bromide, are not known.

There was also a real threat of biological weapons, namely anthrax, plague, and botulinum. The UK programme involved immunisations against plague, with pertussis to accelerate the immune response, and anthrax. The US programme involved immunisations against anthrax and botulinum. Personnel received the vaccinations either before deployment or on arrival in the Gulf theatre. Regular vaccinations in the military are a common practice but immunisation against biological weapons is rare, leading to speculation that multiple vaccinations may have damaged the immune system. Rook and Zumla hypothesised that the regimen of multiple vaccinations given to Gulf veterans during stress, especially the administration of pertussis, may have interacted with pesticides to cause a shift in the $\mathrm{T}$ cell cytokine profiles from Th1 to Th2, which may have led to symptom development ${ }^{13}$ (fig 2 ). Receiving multiple vaccines during deployment but not before deployment was associated with multiple symptoms in UK Gulf veterans and lends some support to this theory. ${ }^{14}$

The evidence to date suggests that there are serious biases that may invalidate the reported associations between exposures and ill health. Most of the exposures have been assessed using self report measures and are now objectively unmeasurable. The alleged exposures were not systemically measured in the Gulf theatre. Potential interactions between various exposures remain poorly understood.

Box 3: Gulf specific exposures
- Using self report measures, a wide range of Gulf specific
exposures have been found to be associated with ill health
- There is insufficient objective evidence for any one expo-
sure
- Long term effects of low doses of postulated toxins, such
as chemical agents, depleted uranium, and pesticides are
uncertain but are unlikely to play an important aetiological
role in the development of symptoms

\section{Role of stress}

The US Presidential Advisory Committee concluded that among other factors, stress was likely to be an important contributing factor to Gulf War related illnesses. ${ }^{11}{ }^{15}$

Similar syndromes have arisen from previous conflicts. These conditions have received many different labels: "soldier's heart and effort syndrome" were borne from the Crimean war, "shell shock" and "neurasthenia" from the first world war, and "Agent Orange syndrome" following the Vietnam war. ${ }^{16}$ These wars were horrific in the toll of human lives and the extreme living conditions. No physical cause was found for the health problems, and the psychological impact of conflict began to be increasingly recognised. Post-traumatic stress disorder emerged as a diagnosable condition following the Vietnam war, in an attempt to recognise the role of extreme stress in the development of certain mental health problems. There is also increasing evidence that physical symptoms and conditions are common in people with post-traumatic stress disorder.

The symptoms in Gulf veterans are similar to symptoms in other conflicts in that they share fatigue, shortness of breath, headache, sleep difficulties, impaired concentration, and forgetfulness. ${ }^{16}$ On first impressions, the Gulf War cannot compare with human casualties and settings of previous conflicts. On closer inspection, Gulf veterans were under considerable stress. The threat of biological and chemical warfare was real, constant, and serious and the associated fear cannot be underestimated. It has been argued that biological and chemical agents are as much psychological as physical weapons. 


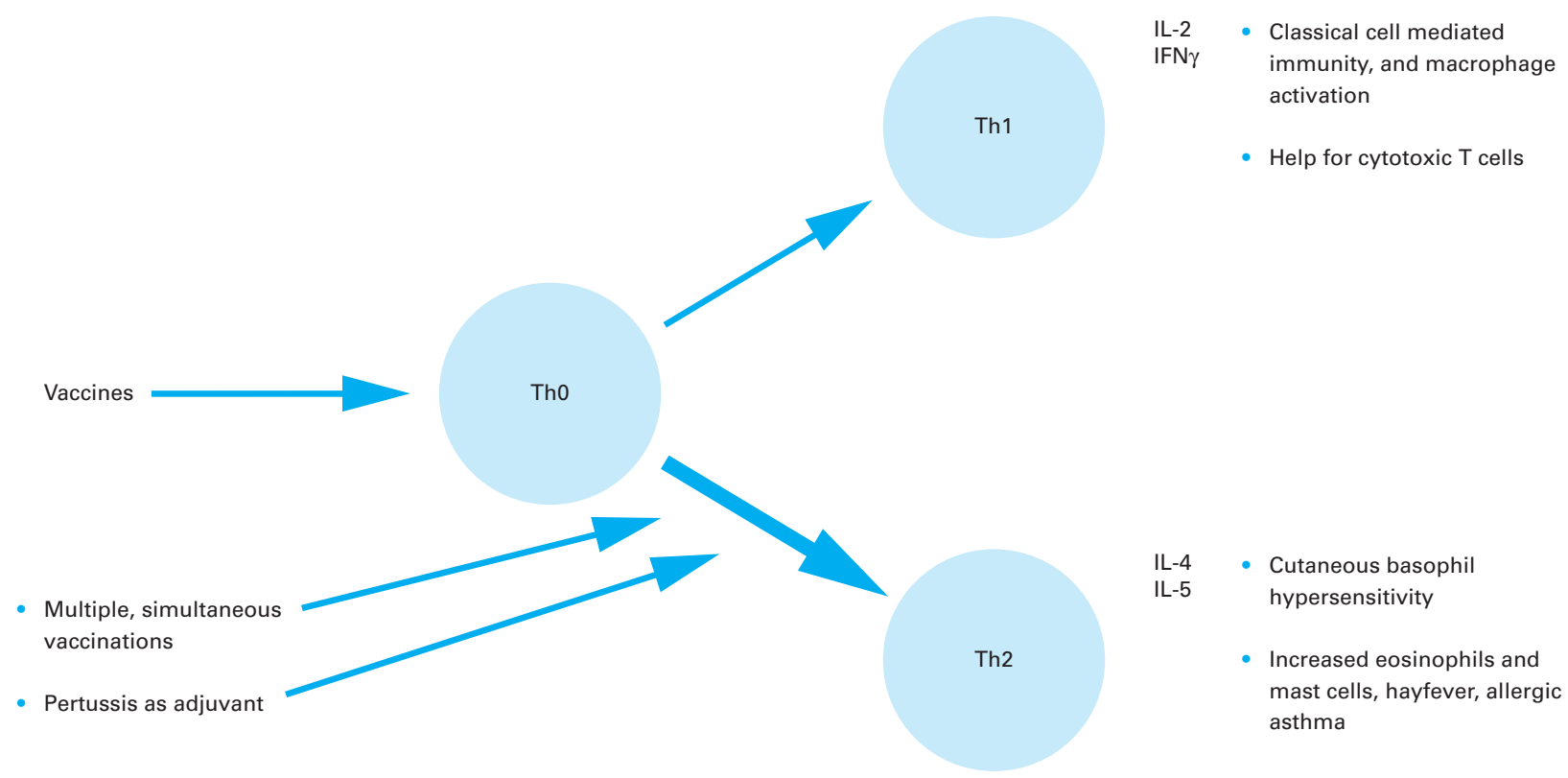

Figure 2 The Rook and Zumula ${ }^{13}$ theoretical model of a potential effect of multiple vaccine on immune function.

Similar syndromes have also been identified in the civilian population. A number of multi symptom, medically unexplained syndromes are very similar to those found in Gulf veterans. These conditions are chronic fatigue syndrome, total allergy syndrome, dental amalgam disease, chronic Lyme disease, sick building syndrome, multiple chemical sensitivity, fibromyalgia, and irritable bowel syndrome. These symptoms and conditions tend to overlap with each other ${ }^{17}$ and are commonly associated with mood. They tend to be similar in that no physical cause can be identified and stressful events are associated with their onset. They tend to differ in the aetiological attribution of the condition given by the patient and the media, from viruses to chemicals to buildings, in the context of inadequate epidemiological or biological evidence for a causal relationship. Elevated rates of chronic fatigue syndrome and multiple chemical sensitivity have been reported in Gulf veterans.

\section{Other explanatory factors}

Sociodemographic risk factors that pertain to many health problems in the civilian populations, such as socioeconomic status and lifestyles, appear to be relevant to ill health in Gulf veterans. Privates were around $20 \%$ more likely to report ill health than non-commissioned officers and around $70 \%$ more likely to report ill health than officers. Ex-service Gulf veterans were around two times more likely to report

\section{Box 4: Non-Gulf specific exposures}

- A number of non-Gulf specific exposures, such as smoking, rank, ex-service, have been associated with ill health in Gulf veterans

- Stress is likely to have played an important role in the aetiology of symptoms

- Symptoms in Gulf veterans appear to have some similarity with symptoms following previous conflicts and in civilian populations psychological and physical ill health, ${ }^{18}$ perhaps because they now adopted a more sedentary civilian lifestyle. Smoking was also associated with ill health. Pre-deployment training, group cohesiveness, such as the 'buddy' system, and post-deployment leave have been considered important in preventing psychiatric breakdown since the second world war. In one tentative survey, there was no evidence that proxy markers of these factors were important for ill health, except perhaps in combat units. ${ }^{18}$

Social and cultural values play a role in the emergence and acceptance of a new disorder. ${ }^{1}$ Prevailing attitudes towards homosexuality interfered with the media and public understanding of HIV and AIDS. Disorders defined by clinical features as opposed to pathology, such as depression and medically unexplained syndromes, are more vulnerable to having their definition distorted by the prevailing attitudes of society and the media. It is difficult to quantify the impact of war images and media information (fig 3) on the onset and appraisal of symptoms in Gulf veterans, but it is possible to speculate that for some Gulf veterans this may have led to attributing non-specific symptoms to their experience in the Gulf. $^{3}$

\section{Treatments}

Interventions have been limited. In the light of no single identified factor or clearly defined disease process to treat, some clinicians have successfully used a multidisciplinary model based on the management of chronic pain. ${ }^{19}$ The model of care would require forming a therapeutic relationship where the experience of symptoms by the veterans is acknowledged by the clinician. This involves a detailed medical and psychiatric assessment and investigations as appropriate to exclude physical and psychiatric explanations for the symptoms. The patient should be encouraged to shift his perception of his condition from one of cause (as no single cause has been identified) to one of rehabilitation, as would be the case with any other chronic condition, such as heart disease. The patient could 


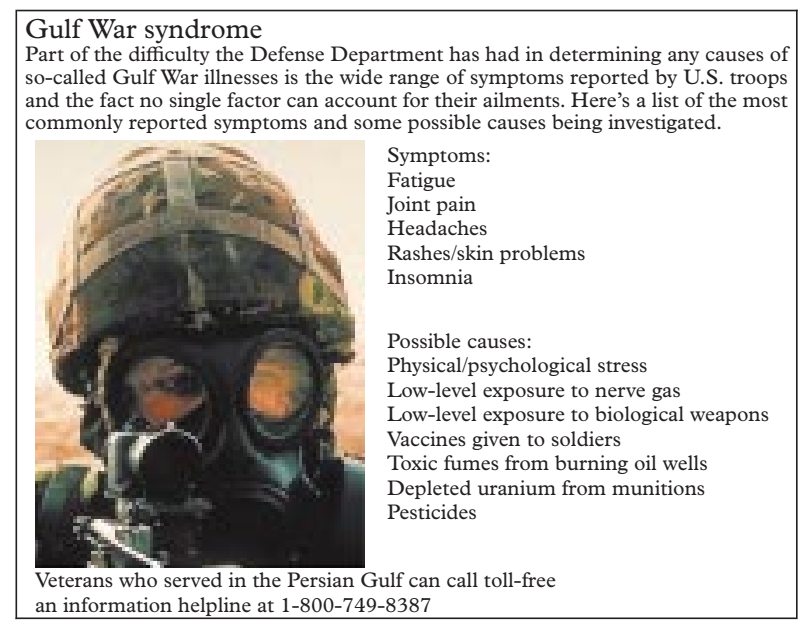

an information helpline at 1-800-749-8387

Figure 3 Example of a US media report (NBC News) on "Gulf War syndrome".

be encouraged to increase his functional capacity by participating in a graded exercise programme that he finds acceptable and promoting strategies that help him to not excessively focus on his symptoms. Any concurrent psychiatric condition-typically depression, anxiety, post-traumatic stress disorder, and alcohol problems-should be actively treated.

\section{Outcome studies}

To date there have been very few outcome studies that examine the course and persistence of symptoms in Gulf veterans, and to monitor the latency period for an emerging illness. In a cohort of US Gulf veterans who were assessed soon after returning from the Gulf, the prevalence of post-traumatic stress symptoms had increased twofold two years later. Post-traumatic stress symptoms were also associated with increased somatic symptoms two years later. A case-control study nested within a randomly selected cohort of US Gulf veterans reported that psychological symptoms and unexplained fatigue tend to persist but other symptoms, such as musculoskeletal pain, gastrointestinal complaints, and skin rashes, fluctuate. These early results suggest that symptoms may have a delayed onset and follow a fluctuating course. Other outcome studies, such as those run by the Gulf War Illnesses Research Unit at King's College, London, are in progress.

\section{Methodological problems}

The study of ill health in Gulf veterans has been limited by both avoidable and unavoidable methodological problems. Studies that use selective samples such as veterans who are in current service or in reserve units have limited generalisability. Studies that do not use a control group cannot make inferences for a new disorder, as they have not tested whether similar problems are present in non-Gulf samples. The use of self report measures, while the most practical tool in large surveys, may risk overestimating health problems, so do need validating by objective assessments.

The time lag between the Gulf conflict and current research activities is over 10 years, suggesting that it is unlikely that any causal inference can be made from self report measures of exposures. Yet there are almost no objective methods of measuring the various exposures. The evidence that some Gulf veterans are still reporting ill health cannot be ignored and aetiological models are still needed.
Perhaps models that attempt to describe the nature of ill health are one way forward. For example, can current ill health be explained by clinician evaluated psychiatric disorders?

Another complication of the time lag is tracing Gulf veterans. Many of them have left the services and put their military experience behind them. Issues relating to confidentiality can affect tracking of current serving and discharged veterans. The sociodemographic characteristics of most military personnel - that is, male, young, mobile, and of lower ranks - makes them a more difficult group to trace.

\section{Box 5: Methodological problems}

- Time lag since Gulf conflict (1990-91) and current health

- Recall bias of Gulf specific exposures

- Need to use study samples that are representative of the population that was deployed to the Gulf

- Appropriate (other military) comparison groups need to be used to test differences in health and exposures

\section{Lessons for the future}

Modern militaries are different to previous militaries: a greater proportion comprises combat support and other support services units and a smaller proportion of combat units; highly advanced technologies are used which are not without their own risks; and peacekeeping and managing human suffering are increasingly playing a role.

Post-conflict health problems are likely to continue to emerge. The main aim of military organisations should be prevention. The mechanisms currently available are risk communication, collection of routine data, and commissioning research.

Risk communication strategies applied to civilian populations may need to be considered by military organisations to develop further their own well established risk communication..$^{20}$ Effective risk communication involves providing a balance between the amount, timing, and setting of dissemination. Different organisations need to consider strategies appropriate to them, but the key components of risk communication involves identifying the risk(s) that need to be conveyed, understanding the target audience(s), selecting the channels and methods by which the messages will be disseminated, implementing the communications, and assessing feedback. In future conflicts personnel could be educated about the physical and mental effects of deployment rather than solely on obvious life events such as combat.

One of the recurrent difficulties in establishing causal links between Gulf specific exposures and subsequent ill health has been the lack of valid exposure data. Future deployments will require planning to measure potential environmental risk factors. Some measures are related to logistics, such as medical record keeping in and out of field hospitals. Pre-deployment standardised examinations will provide baseline health data from which prospective studies can be mounted. Potential environmental risk factors for each conflict zone should be actively sought and measured at baseline.

The initial reluctance of governments to acknowledge a health problem led to conspiracy theories by the media and veterans group. ${ }^{311}$ As public and media concern about specific exposures are unpredictable, military organisations and governments need to be prepared to act swiftly by commissioning research early and setting up evaluation centres. 
Finally, research is still ongoing. In the UK, three population based projects (University of Manchester, King's College, and the London School of Hygiene and Tropical Medicine) continue to generate epidemiological data on aetiology and outcome of UK Gulf veterans. The USA continues to invest into genetic, immunological, toxicology, clinical, and epidemiological research studies and the results will continue to be published for years to come.

Box 6: Future conflicts
- Surveillance and medical record keeping during deploy-
ments should be a key strategy in detecting and prevent-
ing health problems
- Implementing research earlier rather than later should be
encouraged
- Strategies to improve risk communication will become
increasingly important

\section{Conclusions}

Gulf veterans do have increased health problems. At present, they appear to have a clinical profile related to other medically unexplained conditions, such as chronic fatigue syndrome. No single aetiological factor has been identified and it is likely that Gulf war related ill health is caused by a complex interaction of multiple factors, such as stress, vaccinations, and societal factors. The main lesson for the future is to prevent post-conflict health problems by employing multiple approaches, using risk communication, surveillance, and commissioning research. To date, there is no consistent evidence of a syndrome unique to Gulf veterans. The controversy reminds us that militaries are made up of individuals whose occupation is to protect the rights of citizens in their own, and in other nations, often in extreme conditions.

\section{References}

1 Wegman D, Woods N, Bailar J. Invited commentary: How would we know a Gulf war syndrome if we saw one? Am J Epidemiol 1997;146:704-11.

- This is an excellent description of the methodological issues in assessing emerging disorders.

2 Joseph S, and the Comprehensive Clinical Evaluation Program Evaluation Team. A comprehensive clinical evaluation of 20000 Persian Gulf war veterans. Milit Med 1997;162:149-55.

3 Wessely S, Chalder T, Davies K, et al. Ten years on: what do we know about Gulf war syndrome? In: Havenaar J, ed. Health consequences of ecological disasters. (in press)

- This is an exhaustive review of the epidemiological and biological data published to date as well as an opinion on the cultural factors involved in the emergence of new disorders.

4 The lowa Persian Gulf Study Group. Self-reported illnesses and health status among Gulf war veterans. A population based study. JAMA 1997;277:238-45.
5 Fukuda K, Nisenbaum R, Stewart G, et al. Chronic multi-symptom illness affecting Air Force veterans of the Gulf war. JAMA 1998;280:981-8.

6 Gray G, Kaiser K, Hawksworth A, et al. Increased postwar symptoms and psychological morbidity among US Navy Gulf war veterans. Am J Trop Med Hygiene 1999:60:758-66.

7 Unwin C, Blatchley N, Coker W, et al. Health of UK servicemen who served in the Persian Gulf war. Lancet 1999;353:169-78.

- This was the first UK based population study using two control groups. It confirmed earlier US findings and established that there was an increase in symptoms in UK Gulf veterans.

8 Gray G, Coate B, Anderson C, et al. The postwar hospitalization experience of US veterans of the Persian Gulf war. N Engl J Med 1996;335:1505-13.

9 Macfarlane G, Thomas E, Cherry N. Mortality among UK Gulf war veterans. Lancet 2000;356:17-21.

- This is a well designed and implemented UK based population study of the mortality rates in UK Gulf veterans.

10 Ismail $\mathrm{K}$, Everitt $\mathrm{B}$, Blatchley $\mathrm{H}$, et al. Is there a Gulf war syndrome? Lancet 1999;353:179-82.

11 Presidential Advisory Committee on Gulf War Veterans' IIInesses. Final report. Washington, DC: US Government Printing Office, 1996.

- This report is an excellent summary of the evidence of health problems and the exposures. It makes recommendations and reports the opinions of various individuals and organisations. It is an example of how an enquiry should be conducted.

12 Institute of Medicine. Gulf war and health: volume 1. Depleted uranium, pyridostigmine bromide, sarin, and vaccines. Washington DC: National Academy Press, 2000.

- This is part of a series of exhaustive reports summarising Gulf and non-Gulf evidence for the health effects of various exposures. It can be obtained free from the website <http://www.nap.edu>.

13 Rook G, Zumla A. Gulf war syndrome: is it due to a systemic shift in cytokine balance towards a Th2 profile? Lancet 1997;349:1831-3.

- This is a pioneering theoretical paper which proposes how multiple psychological and biological stresses could potentially lead to immune changes.

14 Hotopf M, David A, Hull L, et al. Role of vaccinations as risk factors for ill health in veterans of the Gulf war: cross sectional study. BMJ 2000;320:1363-7.

15 Marshall G, Davis L, Sherbourne C. A review of the scientific literature as it pertains to Gulf war illnesses, volume 4: stress. Santa Monica, California: RAND Health, $2000<\mathrm{http} / /$ rand.org $>$.

- This is an exhaustive review of stress and its health effects using evidence from civilian and military populations. It can be obtained free from the website.

16 Hymans K, Wignall S, Roswell R. War syndromes and their evaluation: from the US civil war to the Persian Gulf war. Ann Intern Med 1997; 125:398-405.

- This is a seminal and highly readable paper reviewing Gulf War health in the context of other post conflict health problems.

17 Wessely S, Nimnuan C, Sharpe M. Functional somatic syndromes: one or many? Lancet 1978;354:936-9.

18 Ismail K, Blatchley N, Hotopf M, et al. Occupational risk factors for ill health in UK Gulf veterans. J Epidemiol Community Health 2000;54:834-8.

19 Engel CCJ, Roy M, Kayanan D, et al. Multidisciplinary treatment of persistent symptoms after Gulf war service. Mil Med 1998;163:202-8.

20 Institute of Medicine. Strategies to protect the health of deployed U.S. forces: medical surveillance, record keeping, and risk reduction. Washington DC: National Academy Press, 1999.

- This is a well written and thoughtful report on various strategies to decrease the likelihood of health problems in future conflicts. It can be obtained on the website <http://www.nap.edu>. 


\section{QUESTIONS (See answers on $p$ 715)}

(1) Which of the following statements about symptoms in Gulf War related ill health is correct?

(a) Symptoms are rarely associated with psychiatric disorders

(b) Symptoms fall into a unique cluster that is different to other disorders

(c) One of the most commonly reported symptom is headache

(d) Symptoms that are common in Gulf veterans are as common in veterans not deployed to the Gulf

(e) As a group Gulf veterans are severely physically impaired

(2) A variety of research methods have been used in the study of Gulf War related ill health. Which of the following methods is most likely to provide data based on samples that are representative of those who served in the Gulf conflict?

(a) Case series

(b) Voluntary registries

(c) Selected military units of personnel in current service

(d) Population based cross sectional studies

(e) Outcome studies

(3) Which of the following is a correct interpretation of the current evidence regarding exposures?

(a) Stress is most likely to be the single most important factor in the development of symptoms

(b) The effects of depleted uranium on brain damage are well known

(c) The levels of pesticides used were sufficiently high to be regarded as toxic (d) It is unlikely that biological and chemical weapons were released

(e) Multiple vaccinations are associated with increased psychological problems

(4) Which of the following statements is correct?

(a) There is good evidence that the media has contributed to the emergence of a "Gulf War syndrome"

(b) Medically unexplained syndromes are increased in Gulf veterans

(c) The more deployments a soldier has the more likely he is to develop health problems

(d) Modern conflicts are less stressful than previous conflicts

(e) Most of the symptoms in Gulf War veterans can be explained by post-traumatic stress disorder

(5) In future conflicts:

(a) One of the objectives in risk communication is to assume that the intended audiences have the same characteristics

(b) Compensation should be given earlier rather than later, even if the medical condition is not yet clearly defined

(c) Routine surveillance of deployed personnel would be a useful method of collecting baseline data

(d) Commissioning research should be delayed until there is clear evidence of a health problem

(e) There is no longer any need to promote group cohesiveness in military units 